\title{
Quercetin reduces obesity-induced hepatosteatosis by enhancing mitochondrial oxidative metabolism via heme oxygenase-1
}

\author{
Chu-Sook Kim', Yoonhee Kwon'1, Suck-Young Choe ${ }^{1}$, Sun-Myung Hong ${ }^{1}$, Hoon Yoo ${ }^{2}$, Tsuyoshi Goto ${ }^{3}$, \\ Teruo Kawada ${ }^{3}$, Hye-Seon Choi ${ }^{4}$, Yeonsoo Joe ${ }^{4}$, Hun Taeg Chung ${ }^{4}$ and Rina Yu ${ }^{1 *}$
}

\begin{abstract}
Background: Obesity-induced hepatic lipid accumulation causes lipotoxicity, mitochondrial dysfunction, oxidative stress, and insulin resistance, and is implicated in non-alcoholic hepatic pathologies such as steatohepatitis and fibrosis. Heme oxygenase-1 (HO-1), an important antioxidant enzyme catalyzing the rate-limiting step in heme degradation, protects against oxidative stress, inflammation, and metabolic dysregulation. Here, we demonstrate that the phytochemical, quercetin, a natural polyphenol flavonoid, protects against hepatic steatosis in obese mice fed a high-fat diet, and that it does so by inducing $\mathrm{HO}-1$ and stimulating increased hepatic mitochondrial oxidative metabolism.
\end{abstract}

Methods: Male C57BL/6 mice were fed a regular diet (RD), a high-fat diet (HFD), and an HFD supplemented with quercetin for 9 weeks. Levels of mitochondrial biogenesis and oxidative metabolic transcripts/proteins were measured by real-time PCR and/or Western blotting. HO-1 transcripts/proteins were measured real-time PCR and/or Western blotting.

Results: Quercetin upregulated genes involved in mitochondrial biogenesis and oxidative metabolism in lipid-laden hepatocytes and the livers of HFD-fed obese mice, and this was accompanied by increased levels of the transcription factor, nuclear erythroid 2-related factor 2 (Nrf-2), and HO-1 protein. The HO-1 inducer hemin and the HO-1 byproduct carbon monoxide (CO) also enhanced hepatic oxidative metabolism in HFD-fed obese mice. Moreover, the metabolic changes and the lipid-lowering effects of quercetin were completely blocked by the HO- 1 inhibitor ZnPP and by deficiency of Nrf-2.

Conclusion: These findings suggest that quercetin stimulates hepatic mitochondrial oxidative metabolism by inducing HO-1 via the Nrf-2 pathway. Quercetin may be useful in protecting against obesity-induced hepatosteatosis.

Keywords: Obesity, Hepatosteatosis, Fatty liver disease, Mitochondrial oxidative metabolism

\section{Background}

Obesity is a major risk factor for non-alcoholic fatty liver diseases (NAFLD), a condition ranging from excess triglyceride accumulation as lipid droplets within hepatocytes (fatty liver/steatosis) to steatohepatitis, which is accompanied by hepatocyte injury and inflammation, cell death, and fibrosis [1] and increases the risk of progression to cirrhosis as well as hepatocellular carcinoma [2]. Obesity-

\footnotetext{
* Correspondence: rinayu@ulsan.ac.kr

1 Department of Food Science and Nutrition, University of Ulsan, Ulsan, 680-749, South Korea

Full list of author information is available at the end of the article
}

induced hepatic lipid accumulation results from uptake of circulating free fatty acids and de novo hepatic lipogenesis, leading to lipotoxicity, mitochondrial dysfunction, oxidative stress, and insulin resistance [1]. In addition, the reduction in mitochondrial oxidative metabolism leads to accumulation of partially oxidized intermediates, which further exacerbates insulin resistance and promotes the development of NAFLD [3, 4]. Hence, improving mitochondrial function and biogenesis in obese conditions might be beneficial in protecting against the development and progression of NAFLD.

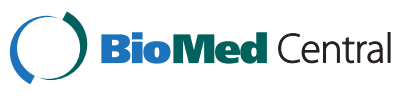

(c) 2015 Kim et al. Open Access This article is distributed under the terms of the Creative Commons Attribution 4.0 International License (http://creativecommons.org/licenses/by/4.0/), which permits unrestricted use, distribution, and reproduction in any medium, provided you give appropriate credit to the original author(s) and the source, provide a link to the Creative Commons license, and indicate if changes were made. The Creative Commons Public Domain Dedication waiver (http://creativecommons.org/publicdomain/zero/1.0/) applies to the data made available in this article, unless otherwise stated. 
Heme oxygenase-1 (HO-1), an important antioxidant enzyme catalyzing the rate-limiting step in heme degradation, is one of factors protecting against oxidative stress, inflammation, and metabolic dysregulation $[5,6]$. HO-1 expression is regulated by the activation of nuclear factor erythroid 2-related factor 2 (Nrf-2), a basic leucine zipper transcription factor [7]. Carbon monoxide (CO), a byproduct of HO-1 activity increases mitochondrial biogenesis by binding to cytochrome c oxidase and stimulating mitochondrial ROS production [8]; it is therefore implicated in mitochondrial oxidative phosphorylation. Moreover, we and others have reported that HO-1 induction by hemin protects against obesity-induced metabolic dysregulation by reducing adipose inflammation [9], which causes mitochondrial dysfunction leading to loss of oxidative capacity and hepatic lipid accumulation $[4,10]$. We therefore hypothesized that dietary factors that promote $\mathrm{HO}-1$ induction and/or Nrf-2 activation might help to maintain hepatic mitochondrial oxidative capacity in obese conditions.

Quercetin (3, 3, 4, 5, 7-pentahydroxyflavone) is a flavonoid that is abundant in various fruits and vegetables and stimulates antioxidant and anti-inflammatory activities [11-14]. We previously showed that it decreased chemokine-induced adipose inflammatory responses by inhibiting the activation of inflammatory signaling molecules [15], and protected against obesity-induced skeletal muscle inflammation [16]. It also reduced ethanolderived oxidative stress in human hepatocytes [17], and stimulated mitochondrial biogenesis in HepG2 cells and lipopolysaccharide-injected mice [18]. It has been suggested that its protective effect is due to $\mathrm{HO}-1$ induction [18]. Quercetin also reduced the expression of genes encoding lipogenic enzymes in rat-liver cells and dietinduced obese mice $[19,20]$. However, its effects on hepatic mitochondrial oxidative metabolism in obese condition remain unclear.

In this study, we demonstrate that quercetin reduces lipid accumulation in primary hepatocytes and the livers of obese mice fed a high-fat diet, and that this is associated with enhanced hepatic mitochondrial oxidative metabolism and $\mathrm{HO}-1$ induction. The metabolic effects of quercetin were blocked by an HO-1 inhibitor and Nrf-2 deficiency. These findings indicate that the beneficial action of quercetin is associated with HO-1 induction through the Nrf-2 pathway. Quercetin may therefore be useful as a dietary additive for reducing obesity-induced hepatosteatosis.

\section{Materials and methods}

\section{Animal experiment}

All animal experiments were approved by the animal ethics committee of the University of Ulsan, and conformed to National Institutes of Health guidelines. Six-week-old male C57BL/6 mice were purchased from Orient Ltd.
(Orient, Busan, Korea). The mice were maintained under specific pathogen-free conditions at $22{ }^{\circ} \mathrm{C}$ and given access to food and water ad libitum.

To examine the effects of quercetin on obesity-induced liver disease, we started the quercetin supplementation together with the establishment of diet-induced obesity. Mice (C57BL/6, male) were adapted for 1 week and then randomly divided into four dietary groups $(n=6$ per group) and fed for 9 weeks on (1) a regular diet (RD) (3.1 kcal/g; $18 \%$ calories from soybean oil; $58 \%$ as carbohydrate; $24 \%$ as protein; Harlan Teklad, Madison, WI); (2) a high-fat diet (HFD) $(5.24 \mathrm{kcal} / \mathrm{g} ; 60 \%$ calories from lard and soybean oil; $20 \%$ as carbohydrate; $20 \%$ as protein; Research Diets, New Brunswick, NJ); (3) the HFD supplemented with $0.05 \%(\mathrm{w} / \mathrm{w})$ quercetin (HFD + $0.05 \%$ Que); and (4) the HFD supplemented with $0.1 \%$ quercetin $(\mathrm{HFD}+0.1 \%$ Que). The dosages of quercetin are equivalent to previous studies $[16,18]$, which are calculated to be $\sim 50$ and $100 \mathrm{mg} / \mathrm{kg}$ body weight per day, respectively.

To evaluate the effect of hemin on obesity-induced liver disease, mice were randomly assigned to the following experimental groups ( $n=5$ per group): (1) the RD + vehicle (RD), (2) the HFD + vehicle (HFD), and (3) the HFD + Hemin. Hemin (Sigma-Aldrich, St. Louis, MO) was dissolved in $0.15 \mathrm{M} \mathrm{NaCl}$ plus $10 \%$ ammonium hydroxide $\left(\mathrm{NH}_{4} \mathrm{OH}\right)$ as a stock solution of $100 \mathrm{mg} / \mathrm{mL}$ and then further diluted $1: 40$ with sterile $0.15 \mathrm{M} \mathrm{NaCl}$. The diluter was intraperitoneally injected (25 mg/kg BW) into the mice three times per week for 2 weeks $[9,21]$. Vehicle-injected mice received an identical $\mathrm{NH}_{4} \mathrm{OH}$ containing solution lacking hemin.

To evaluate the effect of $\mathrm{CO}$, on obesity-induced liver disease, mice were randomly assigned to the following experimental groups ( $n=6-7$ per group): (1) the RD + air (RD), (2) the HFD + air (HFD), and (3) the HFD + carbon monoxide (HFD + CO). Mice inhaled CO (250 ppm) in air (Core Gas Ulsan, Korea) for 2 h (10 AM-12 PM) each day for 10 week. Mice were placed in an exposure chamber (LB science, Daejeon, Korea) at room temperature for exposure to air (control) or to $250 \mathrm{ppm} \mathrm{CO}$ as monitored by a $\mathrm{CO}$ probe (Tongoy Control Technology, Beijing, China) [22].

\section{Isolation of hepatocytes}

Cultures of mouse hepatocytes were prepared as previously described [23]. Briefly, C57BL/6 male mice or Nrf-2 deficient mice (B6/SJL background), 6-8 weeks old, were anesthetized with Nembutal (Dainippon Sumitomo Pharma, Japan) intraperitonealy and their livers were perfused with $40 \mathrm{ml}$ of Liver Perfusion Medium (Gibco, Grand Island, $\mathrm{NY}$ ) followed by $30 \mathrm{ml}$ of Liver Digestion Medium (Gibco), both at a flow rate of $5 \mathrm{ml} / \mathrm{min}$. Hepatocytes were dispersed in Hepatocyte Wash Medium (Gibco) supplemented with $1 \%$ penicillin/streptomycin by dissection and 
gentle shaking. After filtration through a $100 \mu \mathrm{m}$ nylon mesh filter, hepatocytes were purified by repeated centrifugation (three times) at $50 \times g$ for $2 \mathrm{~min}$. A typical yield was about $4-5 \times 10^{7}$ hepatocytes/mouse with $>80 \%$ cell viability as determined by trypan blue exclusion assay. The purity of hepatocytes was determined by flow cytometry (FACSCanto II, BD Bioscience, San Jose, CA). Hepatocytes and non-hepatocytes were separated based on cell size using forward scatter (FSC) and side scatter (SSC). Data were analyzed with Diva Software (BD Bioscience), and $85 \%$ of the cells were found to be hepatocytes. The isolated hepatocytes were resuspended in DMEM (Gibco) supplemented with $10 \%$ FBS, and $1 \%$ penicillin/streptomycin, and cultured in type-1 collagencoated 12 -well plates at a cell density of $2 \times 10^{5}$ cells/well.

\section{Cell culture and treatment of hepatocytes}

To establish lipid-laden hepatocyte, mouse primary hepatocytes on 12-well plates $\left(2 \times 10^{5}\right.$ cells $)$ were treated with 1 mM FFA mixture, a 2:1 ratio of oleate/palmitate coupled to fatty acid-free BSA (molar ratio, 10:1) in DMEM supplemented with $10 \% \mathrm{FBS}$, and $1 \%$ penicillin/streptomycin for $24 \mathrm{~h}$. The cells were exposed to various concentrations of quercetin and/or $0.1 \mu \mathrm{M} \mathrm{ZnPP}$, an HO-1 inhibitor for $24 \mathrm{~h}$ incubation.

\section{Fasting glucose and insulin levels, and glucose tolerance tests}

Plasma insulin levels were determined with the Ultrasensitive Mouse Insulin ELISA (Mercodia, Uppsala, Sweden), and glucose levels were determined with an Accu-Chek glucose monitor and test strips (Roche Diagnostics, Indianapolis, IN). For oral glucose tolerance tests, mice were fasted $12 \mathrm{~h}$ before receiving by oral administration of a $20 \%$ glucose solution at a dose of $2 \mathrm{~g} / \mathrm{kg}$. Blood samples were taken from tail veins at before and 30, 60, 90, and $120 \mathrm{~min}$ after glucose administration and analyzed for glucose levels.

\section{Determination of lipid peroxidation}

Hepatic lipid peroxidation levels were determined by measuring the levels of thiobarbituric acid-reactive substances (TBARS). Briefly, samples were mixed with TBA reagent consisting of thiobarbituric acid (TBA) and $15 \%$ trichloroacetic acid in $0.25 \mathrm{M} \mathrm{HCl}$. The reaction mixture was boiled in a water bath for $1 \mathrm{~h}$ and centrifuged at $2000 \mathrm{rpm}$ for $10 \mathrm{~min}$. The TBARS concentration was determined at $520 \mathrm{~nm}$ absorbance with tetra-methoxypropane as standard. The protein content of homogenates was determined with a BCA protein assay kit (Pierce, Rockford, IL).

\section{Hepatic histology}

Liver tissues were fixed overnight at room temperature in $10 \%$ formaldehyde and embedded in paraffin. Eight micron thick sections were stained with hematoxylineosin and mounted on glass slides. Stained sections were viewed with an Axio-Star Plus microscope (original magnification $\times 200$; Carl Zeiss, Gottingen, Germany).

\section{Hepatic lipid levels}

Hepatic triglyceride content was assayed by saponification in ethanolic $\mathrm{KOH}$, and glycerol content was measured with an FG0100 kit (Sigma-Aldrich) after neutralization with $\mathrm{MgCl}_{2}$. All tissue triglyceride values were converted to glycerol content and corrected for liver weight. Plasma alanine aminotransferase (ALT) level was measured using commercial kits (Asan Pharm. Co., LTD, Gyeonggi-do, Korea).

\section{Real-time PCR}

Total RNA extracted from cultured cells was reverse transcribed into cDNA using M-MLV reverse transcriptase (Promega, Madison, WI). Real-time PCR amplification of the cDNA was performed in duplicate with a SYBR premix Ex Taq kit (TaKaRa Bio Inc., Foster, CA) using a Thermal Cycler Dice (TaKaRa Bio Inc., Japan). All reactions were performed by the same procedure: initial denaturation at $95{ }^{\circ} \mathrm{C}$ for $10 \mathrm{~s}$, followed by $45 \mathrm{cy}$ cles of $95{ }^{\circ} \mathrm{C}$ for $5 \mathrm{~s}$ and $60{ }^{\circ} \mathrm{C}$ for $30 \mathrm{~s}$. Results were analyzed with real-time system TP800 software (Takara Bio, Inc.) and all values for genes were normalized to values for a housekeeping gene. The primers used in the analysis are listed in Table 1.

\section{Western blot analysis}

Samples of $10 \sim 50 \mu \mathrm{g}$ total protein were subjected to western blot analysis using polyclonal antibodies to phosphorylated Akt (Akt-pSer ${ }^{473}$ ), Akt (Cell Signaling, Beverly, MA), HO-1 (Enzo Life Sciences, Farmingdale, NY), COX IV (Abcam, Cambridge, MA), $\alpha$-Tubulin (Abcam), and $\beta$-actin (Sigma-Aldrich). Protein bands were detected using an enhanced chemiluminescence Western blotting detection kit (PerkinElmer, Waltham, MA). Band intensities were quantified by densitometry using Image J program.

\section{Statistical analyses}

Results are presented as means \pm SEM. Statistical analyses were performed using Student's $t$ test. Differences were considered to be significant at $p<0.05$.

\section{Results}

Effect of quercetin on hepatic steatosis and glucose intolerance in obese mice fed an HFD

To examine the effects of quercetin in vivo, we generated obese mice fed an HFD with or without quercetin. Quercetin supplementation significantly reduced hepatic triglyceride concentration in the liver of the HFD-fed 
Table 1 Mouse primers used for real-time PCR analysis

\begin{tabular}{lll}
\hline Gene & Forward primer $\left(5^{\prime} \rightarrow 3^{\prime}\right)$ & Reverse primer $\left(5^{\prime} \rightarrow 3^{\prime}\right)$ \\
\hline Nrf-2 & TCCGCTGCCATCAGTCAGTC & ATTGTGCCTTCAGCGTGCTTC \\
HO-1 & TGCAGGTGATGCTGACAGAGG & GGGATGAGCTAGTGCTGATCTGG \\
PGC-1a & CCGTAAATCTGCGGGATGATG & CAGTTCGTTCGACCTGCGTAA \\
Nrf-1 & GACCTTGCCACAGGCAGGTAA & CGCCTGCTCCATGAACACTC \\
Tfam & TCAGGAGCAGCAGGCACTACA & CTGAGCTCCGAGTCCTTGAACAC \\
PPARa & ACGCTCCCGACCCATCTTAG & TCCATAAATCGGCACCAGGAA \\
CPT-1a & TGGCTTCAGAGCCAGTGGAG & AGCGATGGTGGCTGTCATTC \\
B-actin & CATCCGTAAAGACCTCTATGCCAAC \\
GAPDH & GGCTATCACGGAGGCTGTGAA & ATGGAGCCACCGATCCACA \\
\hline
\end{tabular}

obese mice (Fig. 1a). In agreement with this, lipid deposition in the liver revealed by histochemical analysis was reduced (Fig. 1b). Quercetin also significantly reduced TBARS levels, a marker of lipid peroxidation (Fig. 1c), and ALT levels, a marker of liver damage (Fig. 1d). We further examined whether the metabolic improvement in response to quercetin observed in the livers of the obese mice reduced obesity-induced glucose intolerance. Levels of fasting glucose and insulin were significantly lower by quercetin (Fig.1e). Glucose tolerance tests confirmed that the quercetin-fed obese mice were more glucose tolerant (Fig. 1f), and activation of the insulin

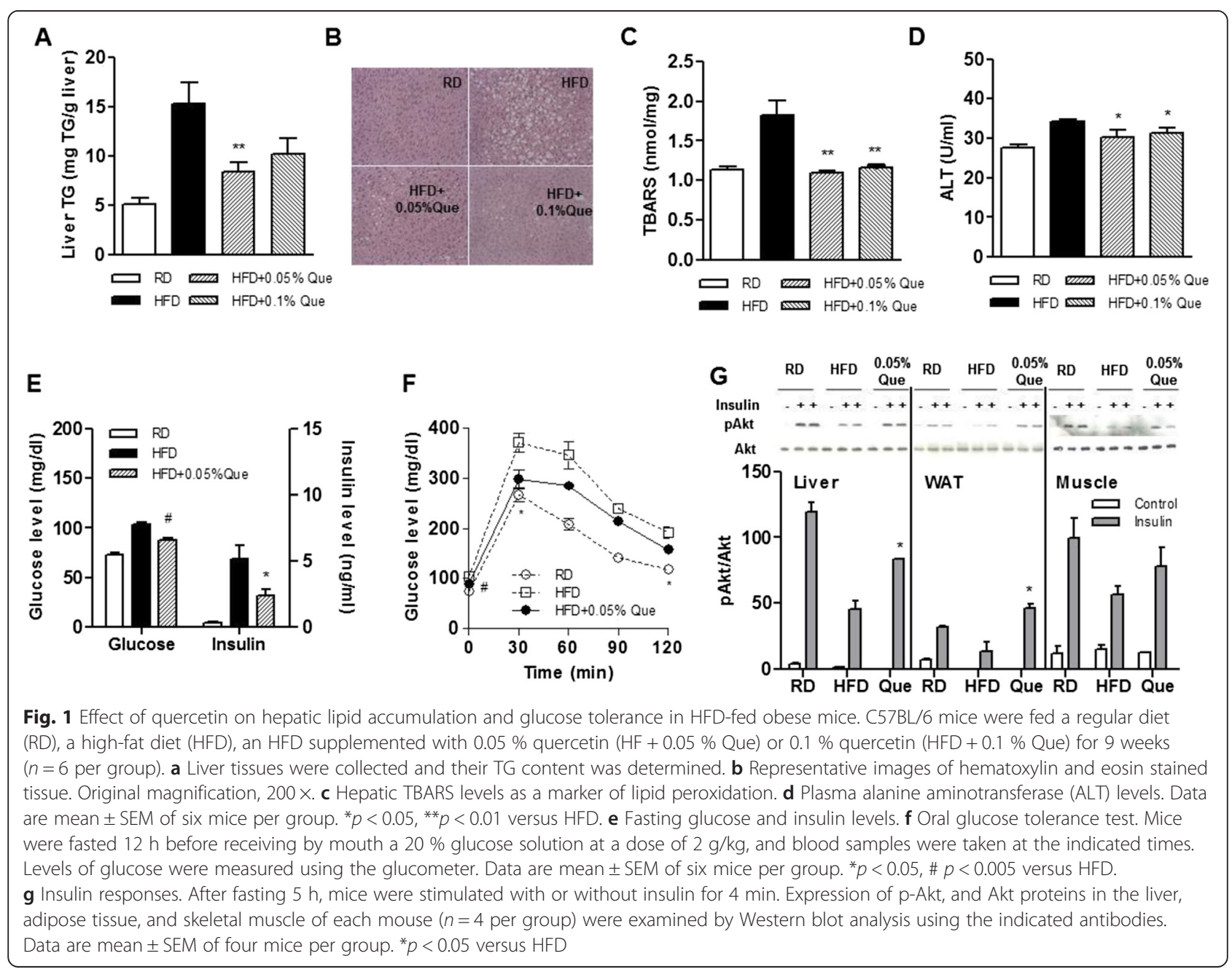


signaling molecules Akt in liver, muscle, and adipose tissue was stimulated (Fig. 1g). Quercetin did not alter food intake, and the HFD-fed mice supplemented with quercetin had a tendency to gain less weight than the control HFD-fed mice (data not shown), as previously reported [16], indicating that the observed benefits of quercetin supplementation are probably associated with the metabolic effects of quercetin.

\section{Induction of HO-1 by quercetin in liver of obese mice fed} an HFD

HO-1 plays an important role in modulating hepatic metabolism by affecting mitochondrial biogenesis and metabolism $[24,25]$. We found that quercetin supplementation significantly upregulated levels of $\mathrm{Nrf}-2$ and $\mathrm{HO}-1$ transcripts (Fig. 2a), and also increased $\mathrm{HO}-1$ protein in the HFD-fed obese mice (Fig. 2b). To see whether it altered mitochondrial oxidative metabolism, we measured markers for mitochondrial biogenesis and oxidative phosphorylation. Levels of PGC-1 $\alpha$ and Tfam transcripts (Fig. 2c), and COX IV protein (Fig. 2d) were indeed also upregulated.

\section{HO-1 inhibition and Nrf-2 deficiency oppose the effects of} quercetin

Using lipid-laden primary hepatocytes mimicking fatty hepatocytes, we tested the idea that quercetin reduces hepatic lipid accumulation by inducing HO-1. As shown in Fig. $3 a$ and $b$, the effect of quercetin on the lipid content of lipid-laden hepatocytes was paralleled by an increase in
A

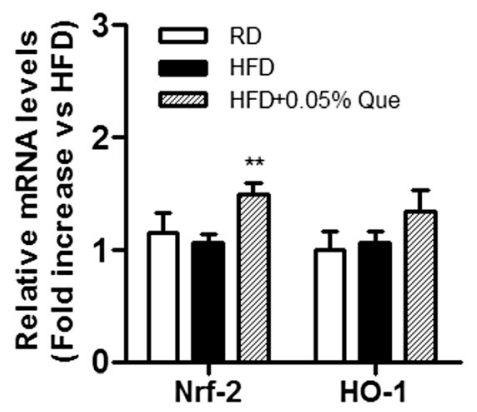

C

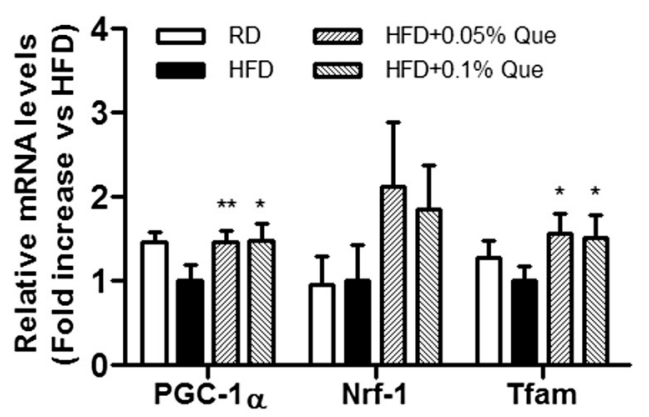

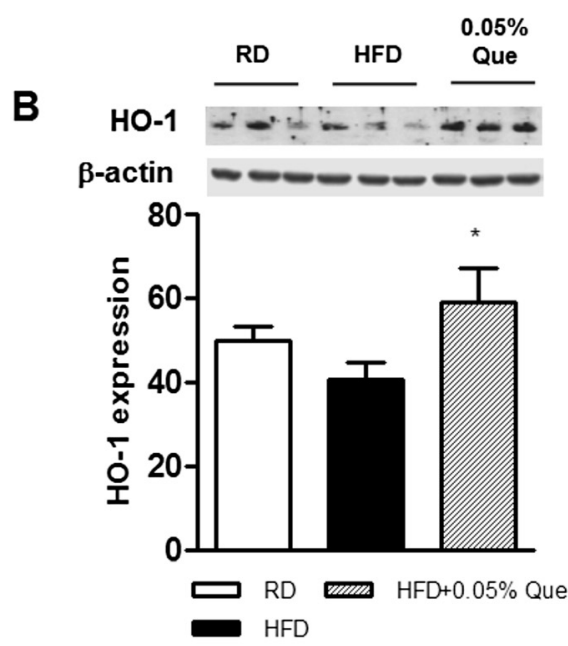

D coX IV 2 RD HFD $\begin{gathered}0.05 \% \\ \text { Que }\end{gathered} \begin{gathered}0.1 \% \\ \text { Que }\end{gathered}$
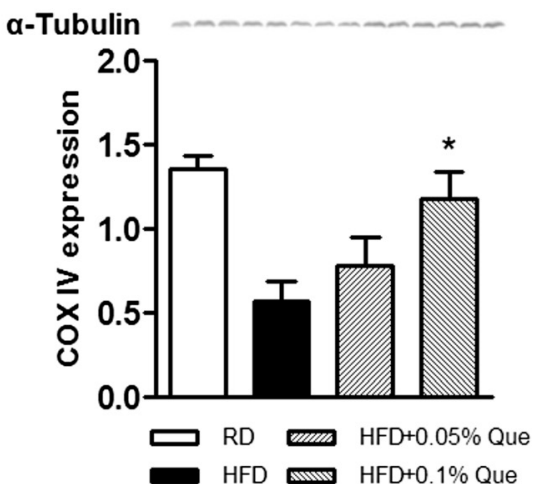

Fig. 2 Effect of quercetin on expression of HO-1 and/or mitochondrial biogenesis markers in HFD-fed obese mice. Livers were isolated from mice fed a regular diet (RD), a high-fat diet (HFD), an HFD supplemented with $0.05 \%$ quercetin (HF + $0.05 \%$ Que) or $0.1 \%$ quercetin (HFD + $0.1 \%$ Que) for 9 weeks ( $n=6$ per group). a Expression of Nrf-2 and HO- 1 mRNAs was quantified by real-time PCR. b Levels of HO- 1 protein were determined by Western blotting. c Expression of PGC-1a, Nrf-1, and Tfam mRNAs was quantified by real-time PCR. d Levels of COX IV protein were determined by Western blotting. Data are mean \pm SEM of six mice per group. ${ }^{*} p<0.05,{ }^{* *} p<0.01$ versus HFD 


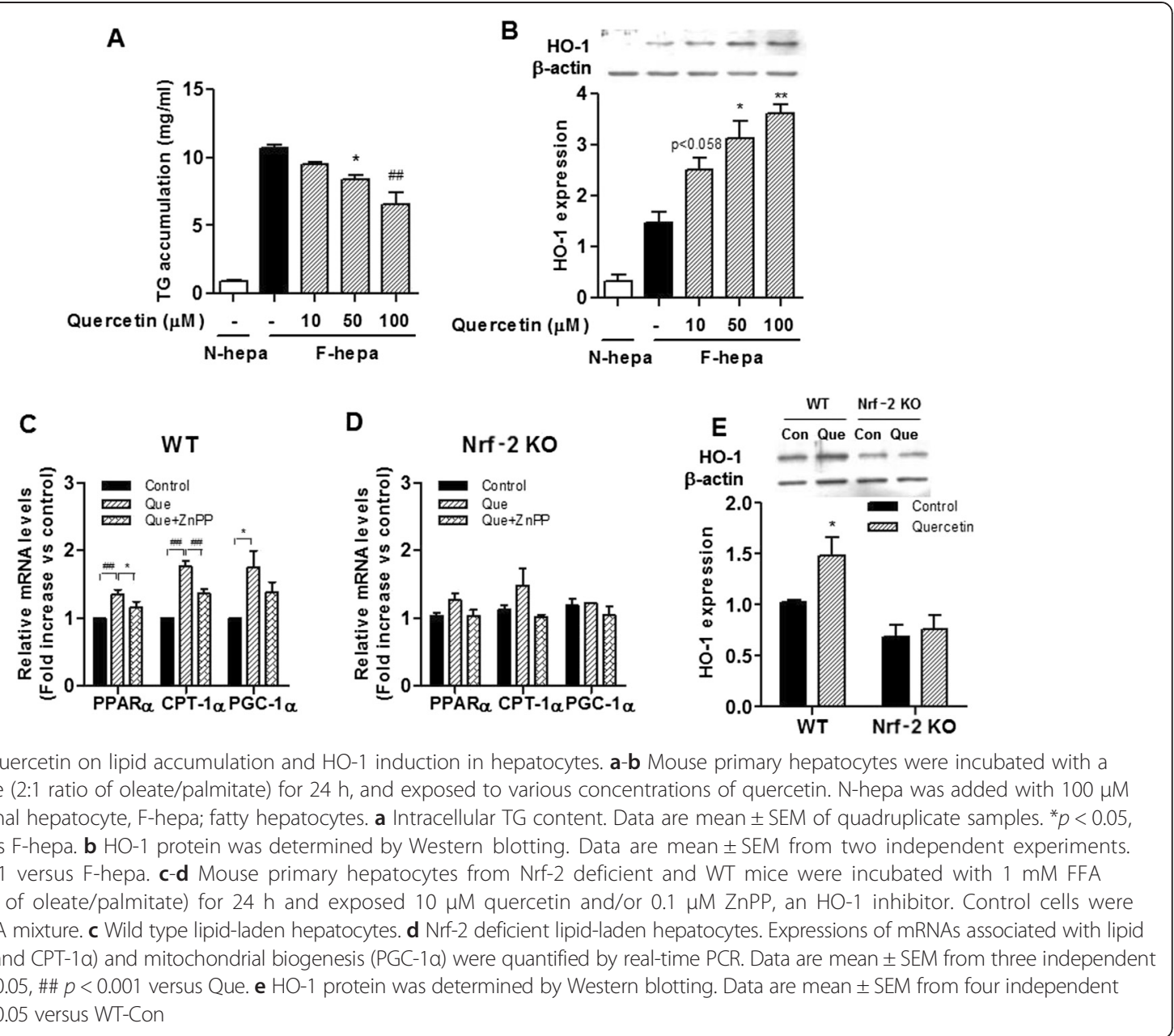

the level of HO-1 protein. Along with this we found that markers of mitochondrial biogenesis such as PGC- $1 \alpha$, and PPAR $\alpha$ and CTP- $1 \alpha$, a marker of mitochondrial oxidative phosphorylation, were upregulated (Fig. 3c). Moreover, $\mathrm{ZnPP}$, a competitive inhibitor of $\mathrm{HO}-1$, significantly reduced these effects (Fig. 3c), and the effects were completely abolished in Nrf-2 deficiency (Fig. 3d). Moreover, quercetin also had no effect on $\mathrm{HO}-1$ protein levels in the Nrf-2-deficient hepatocytes, while it markedly induced HO-1 expression in the WT control (Fig. 3e).

\section{Effect of hemin and carbon monoxide on hepatic mitochondrial metabolism}

We also confirmed the effects of the HO-1 inducer hemin and the HO-1 byproduct carbon monoxide on hepatic lipid content in HFD-fed obese mice. Like quercetin, both treatments reduced hepatic lipid accumulation (Fig. 4a and d) and enhanced markers of mitochondria biogenesis such as Tfam and PGC- $1 \alpha$ transcripts (Fig. $4 \mathrm{~b}$ and e) as well as a marker of oxidative metabolism, COX IV protein (Fig. 4c and f) in the livers of HFD-fed obese mice.

\section{Discussion}

The uptake of circulating free fatty acids together with de novo hepatic lipogenesis can lead to harmful hepatic lipid accumulation. Hence, identifying dietary factors that reduce hepatic lipogenesis and/or increase hepatic lipid oxidation may be useful in reducing hepatic lipid accumulation in obese condition. Quercetin reduces the biosynthesis of hepatic fatty acids and triglycerides in the liver of HFD-fed mice [19]. In this study, we showed that quercetin enhances hepatic mitochondrial oxidative metabolism in lipid-laden hepatocytes and the livers of obese mice fed a high-fat diet, and for the first time demonstrated a causal relationship between the expression of Nrf-2/HO-1, mitochondrial biogenesis and hepatic lipid accumulation using hemin, $\mathrm{ZnPP}$ and $\mathrm{CO}$.

Upregulation of transcripts and proteins involved in mitochondrial biogenesis leads to enhancement of oxidative metabolic capacity. For example, the transcription factor Nrf-1 regulates the transcription of nuclear genes coding for mitochondrial respiratory chain proteins, and it can be activated by PGC- $1 \alpha$, which stimulates the 

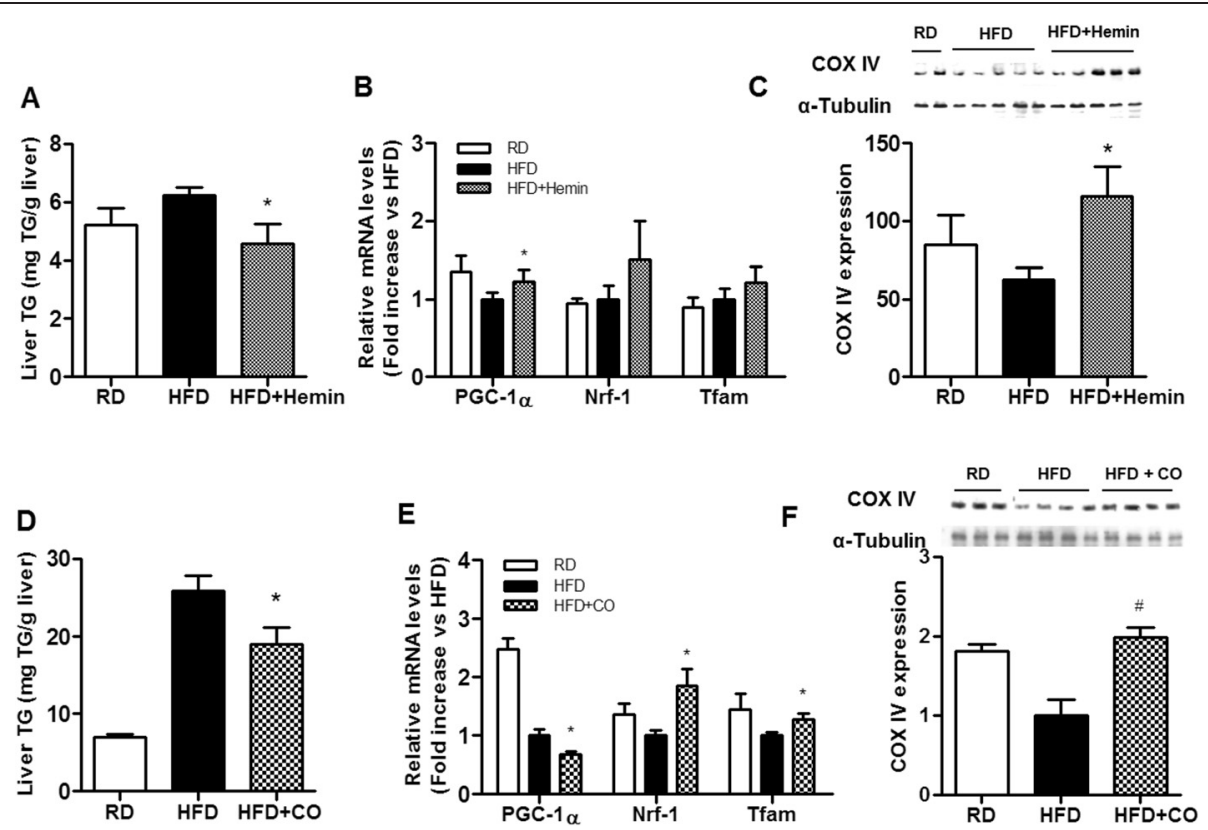

Fig. 4 Effect of an HO-1 inducer and/or CO on hepatic lipid accumulation and mitochondrial oxidative metabolism in HFD-fed mice (a-c) C57BL/ 6 mice were fed an HFD diet for 2 weeks with hemin injection three times per week ( $n=5$ per group). a Liver tissues were collected and their TG contents were determined. b PGC-1a, Nrf-1, and Tfam mRNAs were quantified by real-time PCR. c Expression levels of COX IV protein was determined by Western blotting. Data are mean \pm SEM of five mice per group. ${ }^{*} p<0.05$ versus HFD. $\mathbf{d}$-f C57BL/6 mice inhaled CO ( 250 ppm) for $2 \mathrm{~h}$ each day for 10 weeks ( $n=6-7$ per group). $\mathbf{d}$ Liver tissues were collected and their TG contents were determined. e PGC-1a, Nrf-1, and Tfam mRNAs were quantified by real-time PCR. $\mathbf{f}$ Expression levels of COX IV protein were determined by Western blotting. Data are mean \pm SEM of six to seven mice per group. ${ }^{*} p<0.05$, \# $p<0.005$ versus HFD

transcription of genes involved in oxidative phosphorylation [26, 27]. PGC-1 $\alpha$ and Nrf-1 coactivate the expression of Tfam, which is important for regulating and maintaining mtDNA replication and transcription [26, 27]. Moreover, increases of COX IV typically reflect increased levels of other mitochondrial enzymes of the electron transport chain and enzymes of the $\beta$-oxidation pathway [28]. In this study, we found that quercetin increased transcript levels of genes involved in regulating mitochondria biogenesis such as PGC-1 $\alpha$, Nrf-1, Tfam, as well as COX IV protein in HFD-fed obese mice and in lipid-laden hepatocytes. Others have shown that obesity-induced reduction of transcript of PPAR $\alpha$, which regulates $\beta$ oxidation, is prevented by quercetin supplementation $[19,29]$. Our findings together with these others suggest that quercetin improves hepatic mitochondrial oxidative metabolic capacity by increasing mitochondria biogenesis, and so limits hepatic lipid accumulation. Alternatively, quercetin is a potent scavenger of ROS in many types of cells [30-32], and this may contribute to reduction of mitochondrial oxidative damage and hence maintain its oxidative metabolic capacity. In addition, the reductions in TBARS and plasma levels of ALT indicate that quercetin protects against hepatic mitochondrial damage, which may rescue mitochondrial oxidative capacity.

HO-1 metabolizes heme to biliverdin and releases carbon monoxide and iron [33], and plays an important role in cellular defense against oxidative damage and in cellular metabolism [25]. The transcription factor Nrf-2 is the master regulator of $\mathrm{HO}-1$ gene expression, and the HO-1 product, carbon monoxide, enhances the nuclear translocation of $\mathrm{Nrf}-1$ and $\mathrm{PGC}-1 \alpha$, and activates Tfam expression, all of which augments mitochondrial biogenesis [34, 35]. Studies have shown that quercetin attenuates hepatic lipid accumulation in obese mice fed an HFD [19, 31, 32] and that reduced lipid accumulation is associated with Nrf-2 [31]. Quercetin also increases hepatic mitochondrial biogenesis [18]. However, the link between hepatic lipid accumulation and mitochondrial biogenesis and Nrf-2/HO-1 signaling remained unclear. Interestingly, we observed upregulation of $\mathrm{HO}-1$ protein in the livers of obese mice supplemented with quercetin and in lipid-laden hepatocytes treated with quercetin. Moreover, we found that the HO-1 inducer hemin and the HO-1 byproduct carbon monoxide enhanced levels of transcripts involved in mitochondria biogenesis and oxidative phosphorylation, and reduced hepatic lipid accumulation, indicating that the effect of 


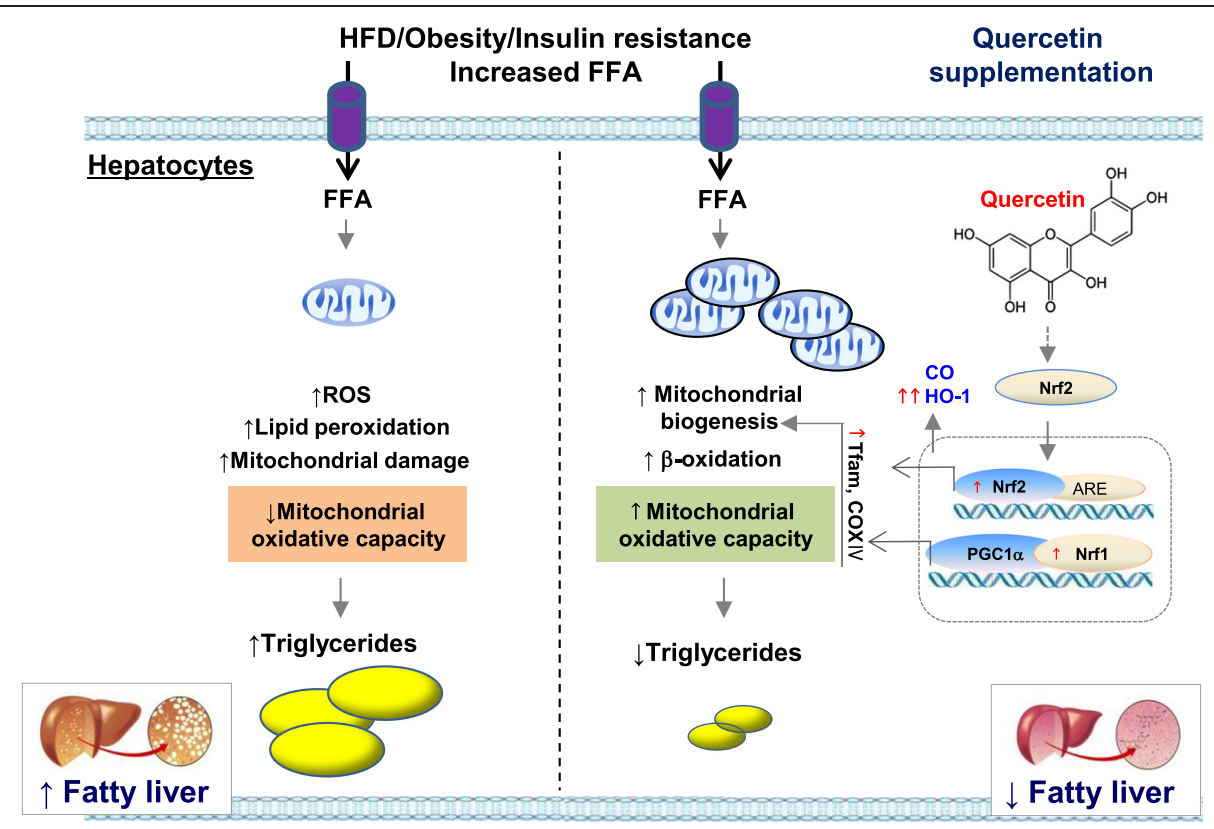

Fig. 5 Proposed mechanism for quercetin action against hepatic steatosis in obese mice. Excess intake of nutrients, including overloaded FFAs in obese conditions, increases ROS production in the liver; the latter results from lipid peroxidation and cause mitochondrial damage. The mitochondrial damage in turn leads to a decrease of mitochondrial oxidative capacity, including $\beta$-oxidation and ATP production, resulting in triglyceride accumulation in the liver. Quercetin limits hepatic lipid accumulation by enhancing hepatic mitochondrial oxidative metabolic capacity. Quercetin increases the induction of $\mathrm{HO}-1$ and its byproduct $\mathrm{CO}$ by the upregulated transcriptional regulator $\mathrm{Nrf}-2$. CO, enhances the nuclear translocation of Nrf-1 and PGC-1a, and activates Tfam expression, all of which augments mitochondrial biogenesis. This leads to reduction of FFAinduced lipid peroxidation, mitochondrial damage and hepatic triglyceride accumulation by increasing mitochondrial oxidative capacity. The effects of quercetin on the induction of $\mathrm{HO}-1$ were completely disrupted in $\mathrm{HO}-1$ inhibitor and/or Nrf-2 deficiency, indicating that one of the mechanisms by which quercetin enhances mitochondrial oxidative metabolism is associated with $\mathrm{HO}-1$ induction through Nrf-2 pathway

quercetin may be due to HO-1 induction. Moreover the beneficial metabolic effect of quercetin on hepatic mitochondrial metabolic markers was abrogated by the HO-1 inhibitor ZnPP in lipid-laden hepatocytes. Quercetin also upregulated Nrf-2, a transcriptional regulator of $\mathrm{HO}-1$, suggesting that quercetin-induced HO- 1 upregulation is mediated by the Nrf- 2 pathway. Indeed, we found that the effects of quercetin on the induction of $\mathrm{HO}-1$ were completely disrupted in Nrf2 deficient lipid-laden hepatocytes, and this was accompanied by a reduction in mitochondrial oxidative metabolic transcripts. These results suggest that one of the mechanisms by which quercetin enhances mitochondrial oxidative metabolism is associated with HO-1 induction through Nrf-2 pathway (Fig. 5).

\section{Conclusion}

In conclusion, quercetin reduced obesity-induced hepatic lipid accumulation by enhancing mitochondrial oxidative capacity, and this was accompanied by induction of HO-1. The action of quercetin was blunted by HO-1 inhibitor and Nrf-2 deficiency, indicating that HO-1 induction by quercetin through $\mathrm{Nrf}-2$ pathway may be among mechanisms contributing to the reduction of obesity-induced hepatic lipid accumulation. Quercetin, an inducer of HO-1, may be useful as a dietary factor for reducing obesity-induced hepatosteatosis.

\section{Abbreviations}

NAFLD: Non-alcoholic fatty liver diseases; Que: Quercetin; RD: Regular diet; HFD: High-fat diet; ALT: Alanine aminotransferase; TBARS: Thiobarbituric acid-reactive substances; HO-1: Heme oxygenase-1; Nrf-2: Nuclear erythroid 2-related factor 2; PPARa: Peroxisome proliferator-activated receptor alpha; PGC-1a: Peroxisome proliferator-activated receptor gamma coactivator-1alpha; CPT-1a: Carnitine palmitoyltransferase-1-alpha; Nrf-1: Nuclear respiratory factor 1; Tfam: Transcription factor A, mitochondrial; COX IV: Cytochrome C oxidase subunit 4; CO: Carbon monoxide.

\section{Competing interests}

The authors declare that there are no competing interests.

\section{Authors' contributions}

CSK participated in design and coordination of the study, collected data and participated in data interpretations, and helped to draft the manuscript. YK collected data and participated in data analysis. YJ and HTC participated in coordination of the animal study, and performed the CO inhalation. SYC, SMH, HY, TG, TK, and HSC reviewed and approved the final manuscript. RY conceived of the study, and participated in its design and coordination, and participated in data interpretations, and drafted the manuscript. All authors read and approved the final manuscript.

\section{Acknowledgements}

This research was supported by the Bio \& Medical Technology Development Program of the National Research Foundation (NRF) funded by the Korean Government (MEST) (2012M3A9C3048687), and by the Basic Science Research Program through the National Research Foundation of Korea (NRF) funded by the Ministry of Education (No. 2011-0014856). 


\section{Author details}

'Department of Food Science and Nutrition, University of Ulsan, Ulsan, 680-749, South Korea. ${ }^{2}$ Department of Pharmacology and Dental Therapeutics, School of Dentistry, Chosun University, Gwangju 501-759, South Korea. ${ }^{3}$ Graduate School of Agriculture, Kyoto University, Uji, Kyoto 611-0011, Japan. ${ }^{4}$ Department of Biological Sciences, University of Ulsan, Ulsan 680-749, South Korea.

Received: 1 July 2015 Accepted: 28 September 2015

Published online: 06 October 2015

\section{References}

1. Koppe SW. Obesity and the liver: nonalcoholic fatty liver disease. Transl Res. 2014; 164:312-22

2. Starley $B Q$, Calcagno CJ, Harrison SA. Nonalcoholic fatty liver disease and hepatocellular carcinoma: a weighty connection. Hepatology. 2010;51:1820-32.

3. Gusdon AM, Song KX, Qu S. Nonalcoholic Fatty liver disease: pathogenesis and therapeutics from a mitochondria-centric perspective. Oxid Med Cell Longev. 2014;2014:637027.

4. Nassir F, Ibdah JA. Role of mitochondria in nonalcoholic fatty liver disease. Int J Mol Sci. 2014;15:8713-42.

5. Ndisang JF. Role of heme oxygenase in inflammation, insulin-signalling, diabetes and obesity. Mediators Inflamm. 2010;2010:359732.

6. Lee TS, Chau LY. Heme oxygenase-1 mediates the anti-inflammatory effect of interleukin-10 in mice. Nat Med. 2002;8:240-6.

7. Alam J, Stewart D, Touchard C, Boinapally S, Choi AM, Cook JL. Nrf2, a Cap'n'Collar transcription factor, regulates induction of the heme oxygenase-1 gene. J Biol Chem. 1999:274:26071-8.

8. Zuckerbraun BS, Chin BY, Bilban M, d'Avila JC, Rao J, Billiar TR, et al. Carbon monoxide signals via inhibition of cytochrome c oxidase and generation of mitochondrial reactive oxygen species. FASEB J. 2007;21:1099-106.

9. Tu TH, Joe Y, Choi HS, Chung HT, Yu R. Induction of heme oxygenase-1 with hemin reduces obesity-induced adipose tissue inflammation via adipose macrophage phenotype switching. Mediators Inflamm. 2014;2014:290708.

10. Alam MA, Rahman MM. Mitochondrial dysfunction in obesity: potential benefit and mechanism of Co-enzyme Q10 supplementation in metabolic syndrome. J Diab Metab Disord. 2014;13:60.

11. Anjaneyulu M, Chopra K. Quercetin, an anti-oxidant bioflavonoid, attenuates diabetic nephropathy in rats. Clin Exp Pharmacol Physiol. 2004;31:244-8.

12. Overman A, Chuang CC, McIntosh M. Quercetin attenuates inflammation in human macrophages and adipocytes exposed to macrophage-conditioned media. Int J Obes (Lond). 2011;35:1165-72.

13. Boots AW, Haenen GR, Bast A. Health effects of quercetin: from antioxidant to nutraceutical. Eur J Pharmacol. 2008;585:325-37.

14. Middleton Jr E, Kandaswami C, Theoharides TC. The effects of plant flavonoids on mammalian cells: implications for inflammation, heart disease, and cancer. Pharmacol Rev. 2000:52:673-751.

15. Noh HJ, Kim CS, Kang JH, Park JY, Choe SY, Hong SM, et al. Quercetin suppresses MIP-1alpha-induced adipose inflammation by downregulating its receptors CCR1/CCR5 and inhibiting inflammatory signaling. J Med Food. 2014;17:550-7.

16. Le NH, Kim CS, Park T, Park JH, Sung MK, Lee DG, et al. Quercetin Protects against Obesity-Induced Skeletal Muscle Inflammation and Atrophy. Mediators Inflamm. 2014;2014:834294.

17. Ye B, Yang JL, Chen LJ, Wu XX, Yang HS, Zhao JM, et al. Induction of apoptosis by phenylisocyanate derivative of quercetin: involvement of heat shock protein. Anticancer Drugs. 2007;18:1165-71.

18. Rayamajhi N, Kim SK, Go H, Joe Y, Callaway Z, Kang JG, et al. Quercetin induces mitochondrial biogenesis through activation of HO-1 in HepG2 cells. Oxid Med Cell Longev. 2013;2013:154279.

19. Jung $\mathrm{CH}, \mathrm{Cho}$ I, Ahn J, Jeon TI, Ha TY. Quercetin reduces high-fat dietinduced fat accumulation in the liver by regulating lipid metabolism genes. Phytother Res. 2013;27:139-43.

20. Gnoni GV, Paglialonga G, Siculella L. Quercetin inhibits fatty acid and triacylglycerol synthesis in rat-liver cells. Eur J Clin Invest. 2009;39:761-8.

21. Nakamichi I, Habtezion A, Zhong B, Contag CH, Butcher EC, Omary MB. Hemin-activated macrophages home to the pancreas and protect from acute pancreatitis via heme oxygenase-1 induction. J Clin Invest. 2005;115:3007-14
22. Zheng M, Zhang Q, Joe $Y$, Kim SK, Uddin MJ, Rhew H, et al. Carbon monoxide-releasing molecules reverse leptin resistance induced by endoplasmic reticulum stress. Am J Physiol Endocrinol Metab. 2013;304:E780-8.

23. Kim Yl, Hirai S, Takahashi H, Goto T, Ohyane C, Tsugane T, et al. 9-oxo-10 (E), 12 (E)-octadecadienoic acid derived from tomato is a potent PPAR a agonist to decrease triglyceride accumulation in mouse primary hepatocytes. Mol Nutr Food Res. 2011;55:585-93.

24. Piantadosi CA, Withers CM, Bartz RR, MacGarvey NC, Fu P, Sweeney TE, et al. Heme oxygenase-1 couples activation of mitochondrial biogenesis to antiinflammatory cytokine expression. J Biol Chem. 2011;286:16374-85.

25. Wegiel B, Nemeth Z, Correa-Costa M, Bulmer AC, Otterbein LE. Heme oxygenase-1: a metabolic nike. Antioxid Redox Signal. 2014;20:1709-22.

26. Hock MB, Kralli A. Transcriptional control of mitochondrial biogenesis and function. Annu Rev Physiol. 2009;71:177-203.

27. Scarpulla RC. Transcriptional paradigms in mammalian mitochondrial biogenesis and function. Physiol Rev. 2008:88:611-38.

28. Davis JM, Murphy EA, Carmichael MD, Davis B. Quercetin increases brain and muscle mitochondrial biogenesis and exercise tolerance. Am J Physiol Regul Integr Comp Physiol. 2009;296:R1071-7.

29. Goto T, Lee JY, Teraminami A, Kim YI, Hirai S, Uemura T, et al. Activation of peroxisome proliferator-activated receptor-alpha stimulates both differentiation and fatty acid oxidation in adipocytes. J Lipid Res. 2011;52:873-84.

30. Hayashi Y, Matsushima M, Nakamura T, Shibasaki M, Hashimoto N, Imaizumi $K$, et al. Quercetin protects against pulmonary oxidant stress via heme oxygenase-1 induction in lung epithelial cells. Biochem Biophys Res Commun. 2012:417:169-74.

31. Chow JM, Shen SC, Huan SK, Lin HY, Chen YC. Quercetin, but not rutin and quercitrin, prevention of $\mathrm{H}_{2} \mathrm{O} 2$-induced apoptosis via anti-oxidant activity and heme oxygenase 1 gene expression in macrophages. Biochem Pharmacol. 2005;69:1839-51.

32. Saw CL, Guo Y, Yang AY, Paredes-Gonzalez X, Ramirez C, Pung D, et al. The berry constituents quercetin, kaempferol, and pterostilbene synergistically attenuate reactive oxygen species: involvement of the Nrf2-ARE signaling pathway. Food Chem Toxicol. 2014;72:303-11.

33. Ryter SW, Alam J, Choi AM. Heme oxygenase-1/carbon monoxide: from basic science to therapeutic applications. Physiol Rev. 2006;86:583-650.

34. Suliman HB, Carraway MS, Ali AS, Reynolds CM, Welty-Wolf KE, Piantadosi $\mathrm{CA}$. The $\mathrm{CO} / \mathrm{HO}$ system reverses inhibition of mitochondrial biogenesis and prevents murine doxorubicin cardiomyopathy. J Clin Invest. 2007;117:3730-41.

35. Piantadosi CA, Carraway MS, Babiker A, Suliman HB. Heme oxygenaseregulates cardiac mitochondrial biogenesis via Nrf2-mediated transcriptional control of nuclear respiratory factor-1. Circ Res. 2008;103:1232-40.

\section{Submit your next manuscript to BioMed Central and take full advantage of:}

- Convenient online submission

- Thorough peer review

- No space constraints or color figure charges

- Immediate publication on acceptance

- Inclusion in PubMed, CAS, Scopus and Google Scholar

- Research which is freely available for redistribution 